\title{
LA TRAUMATIZACIÓN VICARIA EN LOS TRABAJADORES SOCIALES DEL SECTOR JUDICIAL
}

\author{
VICAR TRAUMATIZATION IN SOCIAL WORKERS \\ OF THE JUDICIAL SECTOR
}

\author{
Yoya Betzabé Flores Pérez, \\ Luz Herfilia Cruzado Saucedo ${ }^{2}$
}

\section{RESUMEN}

El presente informe tiene como propósito conocer como la traumatización vicaria afecta y se presenta en los Trabajadores Sociales del sector justicia; investigación que nació de la necesidad de conocer nuevas limitaciones que se presentan al profesional de este sector, siendo la población investigada 100 Trabajadores Sociales que laboral en el sector justicia.

El accionar del trabajador social del sector justicia, se ve limitado por un factor personal, como es la traumatización vicaria, afectando sus emociones y actitudes; el cual es la presentación de síntomas relacionados con estrés postraumático en quienes apoyan a personas afectadas por una experiencia traumática; desarrollándose como resultado de la exposición breve o prolongada, a los traumatismos de la otra persona, combinada con la empatía que el trabajador siente hacia esta persona y con sus propias experiencias dolorosas aún no integradas en su vida.

1 Doctora en Ciencias del Desarrollo Social. Universidad Nacional de Trujillo. La Libertad. yflores@unitru.edu.pe; https://orcid.org/0000-0001-5194-4448

2 Magister en Docencia y Gestión Educativa. Universidad Nacional de Trujillo. La Libertad. lcruzado@unitru.edu.pe; https://orcid.org/0000-0003-2435-616X 
Los métodos que se han utilizado en la presente investigación son el etnográfico, el cual se utilizó in situ, el método inductivo - deductivo permitió describir cómo se presentan las variables de estudio y conocer cómo se presenta la traumatización vicaria, el método analítico sintético utilizado en la etapa de procesamiento y análisis de resultados. El estudio ha puesto énfasis en la aplicación de un cuestionario y entrevista a los Trabajadores Sociales del sector.

PALABRAS CLAVE: Traumatizacion vicaria, justicia.

\section{ABSTRACT}

The purpose of this report is to know how vicarious traumatization affects and occurs in Social Workers in the justice sector; research that was born of the need to know new limitations that are presented to the professional of this sector, being the population researched 100 Social Workers who work in the justice sector.

The actions of the social worker in the justice sector are limited by a personal factor, such as vicarious traumatization, affecting their emotions and attitudes; which is the presentation of symptoms related to post-traumatic stress in those who support people affected by a traumatic experience; developing as a result of brief or prolonged exposure to the traumas of the other person, combined with the empathy that the worker feels towards this person and their own painful experiences not yet integrated into their lives.

The methods that have been used in the present investigation are the ethnographic, which was used insitu, the inductive - deductive method allowed to describe how the study variables are presented and to know how the vicarious traumatization is presented, the synthetic analytical method used in the stage of processing and analysis of results. The study has placed emphasis on the application of a Questionnaire and interview to the Social Workers of the sector.

KEY WORDS: Vicarious traumatization, justice.

\section{INTRODUCCIÓN}

Los Trabajadores Sociales del sector justicia en su labor reflexionan sobre la labor centrada en la acción social, detectando problemáticas de familias en conflictos y el rol que se cumple en las estrategias implementadas con los usuarios, buscando el empoderamiento y la prevención de disfunciones, con la finalidad de bienestar.

El espacio construido por el Trabajador Social judicial es un "espacio de ejercicio profesional definido inicialmente como centrado en el diagnóstico social de situaciones individuales y familiares, paulatinamente fue recuperado o asumiendo, ante la ausencia, imposibilidad o inconveniencia de otras alternativas, la intervención en algunas situaciones que llegaban hasta ellos" (ALDAY: 2004:22). 
Trabajo Social es la disciplina que ocupa de conocer las causas - efectos de los problemas sociales y lograr que los hombres asuman una acción organizada, tanto preventiva como transformadora que lo supere, siendo los puntos centrales desde la postura epistemológica de Trabajo Social:

\section{a) Recontar sus historias}

Se hace referencia a que cuando se encuentra frente a una persona o familia en cualquiera de las situaciones problema, el objetivo principal es comprender lo que pasa en la realidad, elaborar una visión de los problemas que permita ensanchar el campo de las posibilidades.

\section{b) Retraducir sus demandas}

En el accionar del trabajador social predomina el hacer por sobre la reflexión, por lo que se demora mucho tiempo en reconocer la necesidad de centrar la tarea en retraducir el discurso de la otra persona sin ligerezas.

\section{c) Retraducir el problema}

Se parte de señalar que dentro de una sociedad con organizaciones en funcionamiento, es importante que se reconozca la importancia del trabajador social y para que les va a servir este. Al respecto Maturana señala que "disolver el sufrimiento con conversaciones que interfieran con la recurrencia o con el entrecruzamiento de las mismas".

\section{d) Asumir una acción organizada}

Se hace referencia implementar intervenciones concretas coparticipando con los actores, convirtiéndose en un experto de la involucración y participación de los relatos de las familias con las que trabaja de tal forma que entre esta y el profesional se establezca una correspondencia. Este hecho implica un proceso de estar juntos, siendo que la conversación entre la familia y el profesional remite a una búsqueda recíproca de comprensión y a la exploración, de los puntos centrales: la narrativa, el objeto de la demanda y las intervenciones concretas coparticipando con los actores.

El Trabajador Social "opera con actores en una trama de relaciones sociales. Intentamos trabajar con las familias cuyo deseo es de alguna manera tener, hacer, organizar o comprender algún modelo transformador de sus propias vidas" (ALDAY: 2004:36). 
De la misma forma señala Margarita Rosas "es un saber instrumental pues, le permite desenvolverse en su medio, saber que se hace y cómo se hace de acuerdo con la época y las circunstancias particulares" (ROSAS, 1998: 56), por lo que es importante en la intervención saber el sobre qué, para qué, cómo y con quiénes se debe desarrollar la intervención profesional.

Trabajo Social en el campo judicial se vincula estrechamente con la familia, y son considerados como "auxiliares de justicia" (ALDAY: 2004:103), siendo su vinculación con los escritos judiciales a través de los informes sociales.

Por ello, es que se entiende que la familia es la unidad de trabajo y el medio desde donde se atendía y buscaba solucionar la problemática que presentaba una persona y que lo que en los inicios fuera el método de "caso social individual" derivara, en la práctica, en la atención a la familia. La familia en la resolución de conflictos toma un papel básico, debido a que los miembros de la familia tienden a mantener cierta proximidad mutua por el resto de su vida, para bien o para mal, no importa lo que prescriban jueces o terapeutas.

Los mecanismos que generan la entrada a la familia, son diversos y se encuentran desde las diversas fuerzas e instituciones sociales y políticas hasta la legislación ocupando un lugar importante en los tribunales de familia.

Las familias se desenvuelven sobre dos ámbitos el público y el privado, son considerados como espacios diferentes, en los cuales se presentan las conductas de riesgos, siendo los perfiles de las familias multiproblemáticas en las cuales el Trabajador Social debe de llevar a esclarecer sus oportunidades y llevarlas al equilibrio, teniendo un papel importante en los casos que se presentan, siendo los más comunes: tenencia, régimen de visitas, régimen de alimento, divorcio, maltrato, adicciones y procesos de adopción.

El enfoque del Trabajador Social frente a estos problemas es realizar la investigación a fondo a través de técnicas como la historia de vida, conversación, entrevista y observación, teniendo como objetivo posibilitar trascender la demanda superando la idea de que existe un culpable y un inocente, y así retraducirla hacia el reconocimiento de la crisis familiar, teniendo la historia de cada uno de las partes, detectando alianzas, coaliciones, entre los miembros de la familia, logrando que la población tome conciencia de la necesidad de producir modificaciones para lograr un cambio posible que mejore la calidad de vida no sólo de los involucrados. 
$\mathrm{Al}$ inicio de la intervención aporta información sobre la génesis del conflicto, cuáles son los vínculos familiares, cuál es el desempeño laboral y social del individuo, cómo incide la problemática en el medio y en otras áreas de individuo, detección de otros miembros que puedan colaborar. Todo esto basado en la premisa de "reconocer la dignidad de las personas implica aceptarlas tal como son cualesquiera sean sus circunstancias, condiciones, sexo, etnia, cultura, religión, idas, problemas, que presenten individualizándolas en su singularidad como sujetos en relación con otros sujetos, nunca como objetos" (KISNERMAN; 1989: 187).

Posteriormente, investiga la información de las partes y realiza los informes meritorios correspondientes, para que el juez pueda brindar el dictamen o veredicto. Evalúa el proceso judicial realizando la evaluación determinando las posibilidades de cambio de los miembros de la familia y la identificación de la red familiar, siendo un nexo que ayuda a la circulación de la información y a su vez decodifica a los miembros de la familia los alcances o implicaciones de la intervención judicial.

A los Trabajadores Sociales del sector justicia, en especial por los casos en los que interviene, puede sufrir de traumatización vicaria o desgaste por empatía, entendida como "el síndrome que presentan los Trabajadores Sociales que laboran con sobrevivientes de la violencia y/o de situaciones alarmantes, lo cual surge efecto trayendo pesadillas, pensamientos intrusivos, sensación de estar viviendo la experiencia traumática, reacciones fóbicas, apatía generalizada, hipervigilancia, sentimiento de culpa, depresión y diversos signos de aumento de la activación" La clave de la labor del Trabajador Social es realizar un trabajo en red, basándose en que "los actores sociales de toda comunidad aprendimos de ellos que la única manera de cosechar lo que se espera para mejorar es tejiendo una buena red social que no sólo nos permita crecer si no también que nos sostenga cuando pasamos por malos momentos"(ALDAY: 2004:207).

El escuchar uno de estos problemas produce cambios, y para mantener esta situación de manera equilibrada el primer paso es ser consiente que está existiendo ese síndrome en uno mismo, siendo "afectados por emociones, actitudes, necesidades, formas de acción, comunicación, cambios, personalidades, entre otras" (CANTU, 1999: SP).

Ante estas situaciones, los profesionales tienen estrategias de defensa, que según Marcelo Packman, permiten no involucrarse en el problema y no sufrir de la traumatización vicaria, y busca: "insensibilizarse ante el problema, tomando una distancia máxima con el sufrimiento de los individuos, limitar la intervención en térmi- 
nos de técnicas, tratar el problema desde la dimensión política y elegir determinadas familias para seguir el tratamiento". (PACKMAN, 1997: 246)

Estas estrategias de defensa permiten que el profesional mantenga el statu quo en las instituciones, impidiéndoles crear modelos o programas más adecuados a la comunidad a la que están dirigidas.

La traumatización vicaria es un trastorno de estrés, consecuencias normales de vivir una situación anormal; así, en el caso de las víctimas de violencia intrafamiliar que se mantienen en un estado continuo de alerta, miedo y vigilancia frente a un peligro inminente, verán agotadas sus capacidades adaptativas; los síntomas relacionados al trastorno de estrés postraumático enfrentado por las propias víctimas pueden desencadenarse en los prestatarios debido a la fuerte carga traumática ocasionada durante la atención en tanto se da una reiterada confrontación con la violencia, el abuso y el dolor humano.

La traumatización vicaria, se caracteriza por una serie de síntomas que pueden incluir pesadillas recurrentes, pensamientos intrusivos, sensación de estar viviendo la experiencia traumática, reacciones fóbicas, apatía generalizada, sentimiento de culpa, depresión y diversos signos de aumento de la activación (dificultad para conciliar el sueño, irritabilidad, reacciones de sobresalto).

Los síntomas de la traumatización vicaria son:

\section{a) Re experimentación de los acontecimientos traumáticos:}

Manifestados a través de pesadillas, sueños y recuerdos recurrentes e intrusos; ello ocasiona a su vez temores, malestar, angustia. Además, ante cualquier estímulo interno o externo que simbolicen o recuerden el episodio traumático, la persona sentirá un intenso malestar psicológico, así como respuestas de tipo fisiológico.

\section{b) Evitación y embotamiento de la reactividad general:}

La persona realizará un gran esfuerzo por evitar sentimientos, pensamientos y/o conversaciones relacionadas al evento traumático; también evitará actividades, lugares o personas que le recuerden el traumatismo, es característica la disminución del interés o participación en actividades significativas y aparecerán sentimientos de desapego o enajenación de los otros. 


\section{c) Estado de alerta incrementado:}

Frecuentemente la persona tendrá dificultades para conciliar o mantener el sueño.

\section{d) Irritabilidad o explosiones de ira.}

\section{e) Tendrá dificultades para concentrarse.}

Se mantendrá además en estado de hipervigilancia, presentando respuestas exageradas de sobresalto.

Las consecuencias de la traumatización vicaria son diversas, pero en especial son estado permanente de ansiedad, miedo, sentimientos de culpa, frustración, impotencia, actitudes de irreverencia, estado de depresión, deterioro de las relaciones interpersonales, insatisfacción, bajo desempeño laboral, disocian la escucha, extrema preocupación por su propia seguridad, profunda tristeza al tener la convicción de no ser un buen profesional.

La clave de la labor del Trabajador Social es realizar un trabajo en red a través del trabajo interdisciplinario, basándose en que "los actores sociales de toda comunidad aprendimos de ellos que la única manera de cosechar lo que se espera para mejorar es tejiendo una buena red social que no solo nos permita crecer si no también que nos sostenga cuando pasamos por malos momentos" (ALDAY: 2004:136)

Por lo que la intervención del Trabajador Social debe ser articuladora focalizada en la satisfacción personal de los individuos y sus familias, en los procesos que se presenten y basándose en dos salidas el sistema de valores que posee y el manejo teórico sobre el tema.

\subsection{PROBLEMA}

¿Cuál es el factor personal de mayor incidencia que dificulta el ejercicio profesional de las Trabajadores Sociales del sector justicia del Departamento de la Libertad en el 2014?

\subsection{HIPÓTESIS}

El factor personal de mayor incidencia que dificulta el ejercicio profesional de las Trabajadores Sociales del sector justicia del Departamento de La Libertad en el 2014 es la traumatización vicaria. 


\section{MATERIAL Y MÉTODOS}

La población de estudio estuvo constituida por los Trabajadores Sociales del Sector Justicia de la Libertad siendo un total de 100 profesionales.

Los Métodos utilizados en el presente estudio fueron:

- Método Etnográfico: describió las variables de estudio.

- Método inductivo - deductivo: describió las variables de estudio y explicar la traumatización vicaria en los Trabajadores Sociales del sector justicia.

- Método analítico - sintético: utilizado en la etapa de procesamiento y análisis de resultados, facilitando la identificación de la traumatización vicaria en los profesionales.

Las técnicas son observación, entrevista, encuesta; y los instrumentos son Registro y Guía de observación, Registro y Guía de entrevista y el Cuestionario.

\section{RESULTADOS Y DISCUSIÓN}

Dentro del sector justicia se considera al Trabajo Social, según María Angélica Alday como: "la disciplina que se ocupa de conocer las causas - efectos de los problemas sociales y lograr que los hombres asuman una acción organizada, tanto preventiva como transformadora que la supere" (ALDAY: 2004:35)

La labor del trabajador social del sector justicia se centra en el tratamiento de casos sociales y es un proceso metodológico, el cual Margarita Rozas Pagazza, lo define como: "conjunto de procedimientos que ordenan y dan sentido a la intervención, pero fundamentalmente como una estrategia flexible que articula la acción específica de trabajador social con el contexto" (ROSAS, 1998:45).

La profesión de Trabajo Social, es considerada una profesión de mujeres, como lo señala la autora Alicia Genolet en su Libro "La Profesión de Trabajo Social icosa de mujeres? señala que "La profesión de trabajo social se considera que es una labor de mujeres en su mayoría, porque se trabaja con roles asistenciales, promocionales y preventivos, lo cual hace una labor más humanitaria" (GENOLET, 2005:23); lo cual se evidencia en la figura 1 donde se aprecia los datos de los Trabajadores Sociales del sector justicia según edad y sexo evidenciando que de 25 a 30 años el $8 \%$ son mujeres, de 31 a 40 años se observa el 34\% son de sexo femenino y el $1 \%$ de sexo masculino, de 41 a 50 años el $53 \%$ son de sexo femenino, y el $1 \%$ de sexo masculino, de 51 a 60 años, se el $2 \%$ de sexo femenino, de 61 a más se 
observa el 1\%, siendo que en total se aprecia que el $98 \%$ son mujeres y solo el $2 \%$ son hombres.

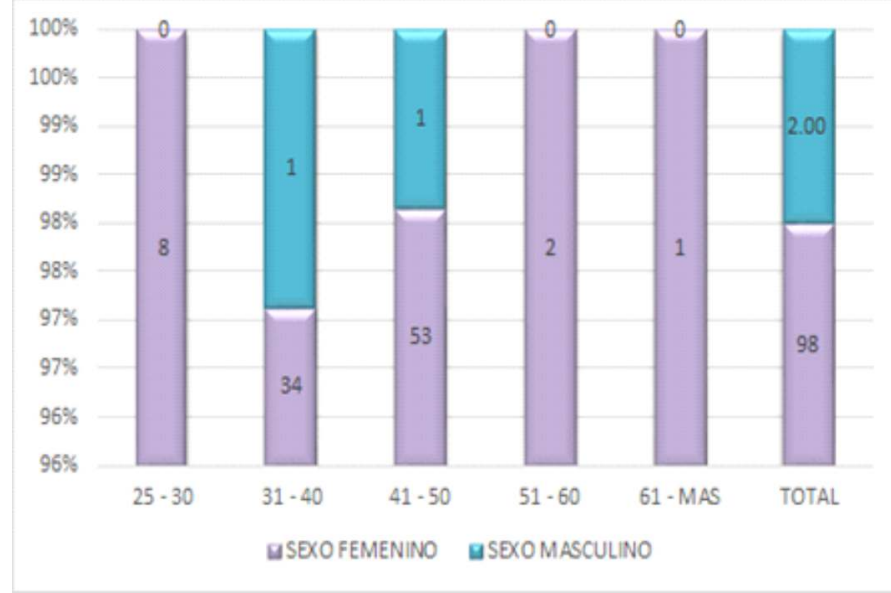

Figura 1. Distribución porcentual de los trabajadores sociales del sector justicia según edad y sexo.

Los datos anteriores se vinculan con las acciones que prioritariamente realizan los Trabajadores Sociales, sin embargo, es necesario conocer el procedimiento para el tratamiento con casos sociales, debido a que es la labor prioritaria que realizan las profesionales de Trabajo Social en este campo de acción, ya que algunos profesionales priorizan diversos pasos dentro del proceso.

Todo esto se evidencia en la figura 2, donde se observa que las acciones que priorizan los Trabajadores Sociales en su labor son el retraducir el problema con un $32 \%$ en mayoría, el asumir una acción organizada un $28 \%$, el retraducir sus demandas con un $25 \%$, el recontar sus historias con un $14 \%$ y planificar sus acciones con un $1 \%$ de manera minoritaria.

El retraducir el problema es poder focalizar y entender la verdadera problemática, focalizándola como punto de partida para un análisis mejor.

Estos elementos básicos conllevan a analizar epistemológicamente la profesión desde una mirada sistémica, y fijar que se trabaja con actores en una trama de relaciones sociales, en las cuales las relaciones familiares son base y cimiento para un futuro mejor, donde el profesional social debe buscar el bienestar encaminando las acciones a partir de las herramientas de las familias. 
Cuando se hace referencia a recontar sus historias, concretamente se refiere a cuando hay un contacto frente a una persona o a una familia en cualquiera de las situaciones problema, el objetivo principal no es comprender lo que pasa en realidad, sino elaborar una visión de los problemas que permitan ensanchar el campo de sus posibilidades.

El retraducir sus demandas es que predomina la acción por reflexión, por lo que se traduce en la necesidad de retraducir el discurso sin ligerezas y responder el pedido del otro sin mayor reflexión.

El retraducir el problema es conocer que el problema pasa en una sociedad que tiene diferentes problemas siendo los contextos ambiguos, y el asumir una acción organizada, es implementar intervenciones concretas coparticipando con los actores. Estos escenarios de crisis familiar se plantean en un espacio de postmodernidad, con una reflexión sistemática, reformulando la práctica que es parte de la vida cotidiana.

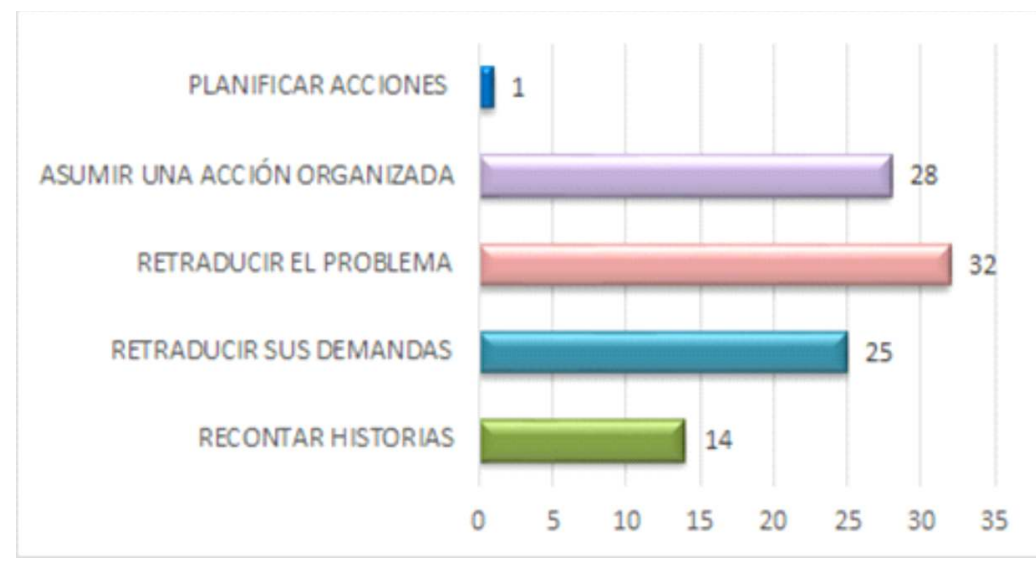

Figura 2. Distribución porcentual de los trabajadores sociales del sector justicia según acciones que prioritariamente realizan.

Estos elementos permiten que el Trabajador Social comprenda la situación de crisis por la que atraviesan las familias, trabajando las relaciones vinculares dentro de esa familia y hacia el contexto social que la rodea, construyendo redes sociales facilitadoras para lograr la superación de la crisis a las que se enfrentan.

Muchas veces la labor se ve dificultada porque algunos profesionales priorizan: 
a) Evitar dedicar el encuentro a la recolección de datos vaciados de significación de hechos dolorosos.

a) La causalidad se basa en la interacción humana tiene una causalidad circular y no lineal.

b) Se conciben a las relaciones conflictivas como interpersonales.

c) Entender que la negociación de los problemas y los ataques a aquellos que los señalan o que intenten enfrentárselos van unidos.

d) Las familias son frágiles, pero en realidad son resistentes y robustas, por lo que no debe preocuparnos la influencia que ejerzamos sobre ellas.

e) La familia es un todo, a veces no entendida de esta forma.

f) Existe el supuesto social de que uno debe salvar a su propia familia.

La labor del Trabajador Social muchas veces se ve muy afectada porque los profesionales se involucran de tal manera, que interiorizan la situación problema, como si fuera propia. Cuando sucede eso, el trabajador social se siente mal, en algunas oportunidades se ha identificado tanto que llora y no quiere volver a ver y seguir con el tratamiento de la familia que está llevando.

Sobre lo que se investiga son situaciones en las que se interviene, abarcando el problema, sus relaciones y conexiones con las distintas dimensiones de la vida social de los sujetos y por las que se busca satisfacer las necesidades.

Muchas veces el profesional sobre las situaciones problemas que investiga las identifica como situaciones cercanas que atravesamos en lo individual, reconociendo que tenemos un vacío en alguna etapa de nuestra vida.

Cuando el trabajador social se inserta en una institución judicial, sabe que se va a enfrentar con situaciones sociales, protagonizadas por sujetos concretos que expresan mayor o en menor medida sufrimiento, odio, molestia, enojo, ira. Por lo que la relación es de extrema cercanía, insertándose de una u otra manera en la vida cotidiana de los usuarios.

Todo ello se observa en la figura $\mathrm{N}^{\circ} 03$ se observan los datos de los Trabajadores Sociales del sector justicia según el factor personal que dificulta su labor profesional, en mayor número se encuentra la identificación con los problemas de las personas con un 48\%, el no manejar técnicas e instrumentos para consejería con un $32 \%$, el no conocer el tratamiento de los casos con un $12 \%$ y el no contar con tiempo para la atención representado con un $8 \%$. 
Yoya Betzabé Flores Pérez, Luz Herfilia Cruzado Saucedo

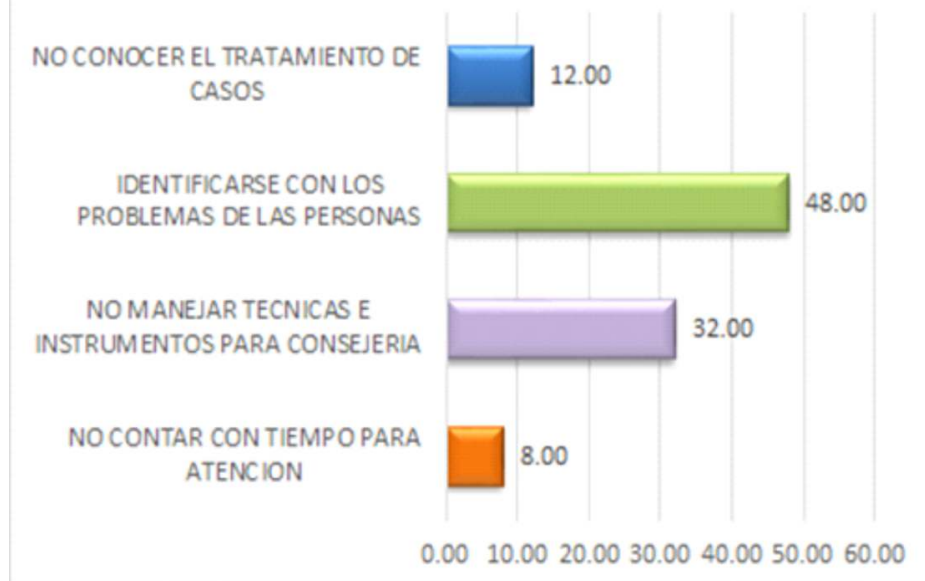

Figura 3. Distribución porcentual de los trabajadores sociales del sector justicia según el factor personal que dificulta su labor profesional.

Un Trabajador Social se identifica con sus casos, muchas veces de manera estrecha, rompiendo la labor profesional, sino estableciendo una relación horizontal, lo cual no cumple el proceso metodológico del profesional.

Todo esto permite rescatar el concepto de "saberes cotidianos", lo cual tiene que ver con una objetivación de los procesos que son subjetivos por medio de los cuales cada individuo construye su propio mundo y muchas veces se parcializan con el mismo individuo. Al respecto Margarita Rozas señala que el saber instrumental es: "permite desenvolverse en su medio, saber qué se hace y cómo se hace de acuerdo con la época y las circunstancias particulares” (ROSAS, 1998:43)

Este concepto debe ser aprendido y aplicado por el Trabajador Social al análisis de la situación concreta, dejando de lado lo subjetivo, basándose sólo en lo objetivo; es decir las situaciones sociales no son a priori, son desencadenantes. Al respecto, María Angélica Alday señala: "ser objeto de los problemas que se toman por objeto" (ALDAY, 2004: 44)

Se observa que muchos Trabajadores Sociales conocen y definen la traumatización vicaria como parte de esta labor que cumplen, siendo un síndrome que presentan los operadores que trabajan con sobrevivientes de violencia, y que pasan por un proceso de atención el cual denominamos el seguimiento de casos sociales, donde el profesional escucha el trauma de lo que pasó, y siente como suyo el problema, comienza a presentar cambios, y mostrar culpa, impotencia, depresión y diversos signos de aumento de la activación. 
Esta situación llega, cuando ambas personas (trabajador social y usuario) se ven afectadas mutuamente por sus emociones y actitudes, necesidades, formas de control, comunicación, y cambio de personalidad; muchas veces, en la mayoría de los casos, el trabajador social desconoce que padece de traumatización vicaria, pero presenta los síntomas, y piensa que es parte de su sensibilidad.

Todo ello se observa en la figura 4 se plasman los datos de los Trabajadores Sociales del sector justicia según el conocimiento sobre la traumatización vicaria evidenciando que el $77 \%$ conoce sobre el tema, mientras que el $23 \%$ no conoce. Del 77\% que conoce la traumatización vicaria se colocó opciones de definiciones teniendo:

A: Identificarte con el problema de la otra persona y no saber cómo actuar, encontrando que un $8 \%$ señala esta opción.

B: Ansiedad y miedo ante los usuarios que requieren la atención, un 46\% señala esta opción.

C: Absorber los problemas de las personas a las cuales atendemos, un $12 \%$ señala esta opción.

D: Presentar irritabilidad y temor a situaciones imprevistas con el usuario, un $11 \%$ señala esta opción.

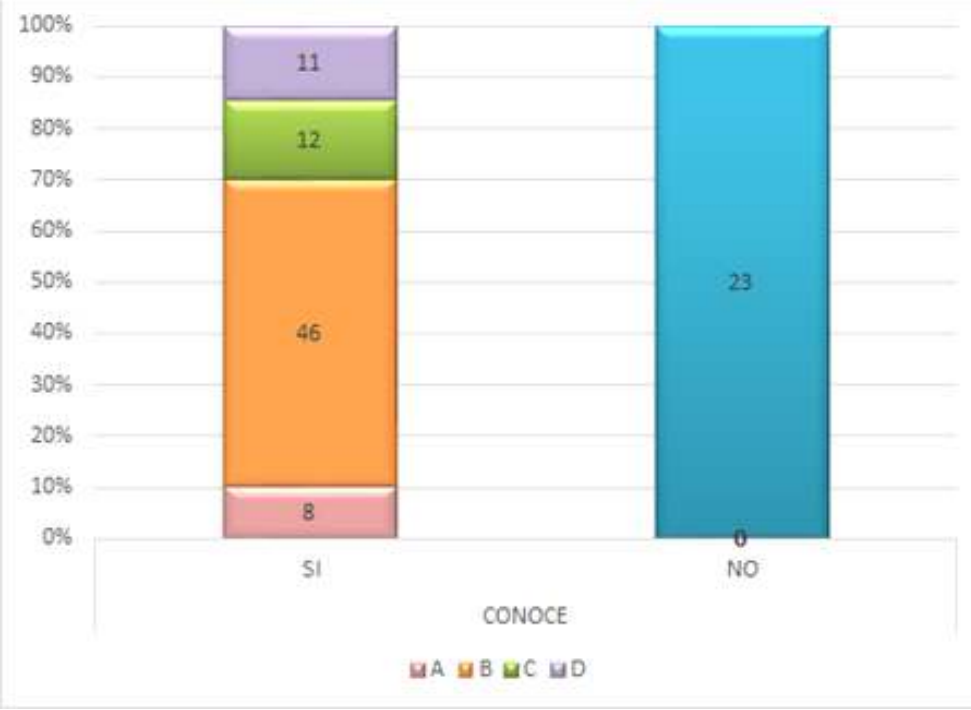

Figura 4. Distribución porcentual de los trabajadores sociales del sector justicia según conoce y define la traumatización vicaria. 
Los Trabajadores sociales de los juzgados optan por alguna estrategia de defensa, como no querer seguir con el tratamiento del caso, derivarlo a otro colega, esconderse en el anonimato, miedo y temor de sufrir la traumatización vicaria, los mecanismos de defensa ante ello son:

a) Con el constante aprendizaje de las nuevas teorías sobre tratamiento de casos.

b) Constante enseñanza de futuros profesionales sobre ello (prácticas pre profesionales).

En la figura 5 se muestran los efectos de la traumatización vicaria en los Trabajadores Sociales encontrando que el 32\% señala que prefiere no atender el caso, $30 \%$ señala que trabajan cada caso de manera personalizada, el 18\% señala que no sabe qué solución darle, el 11\% no quiere dar la cara a la otra persona y un $9 \%$ señala que no quiere revisar el caso.

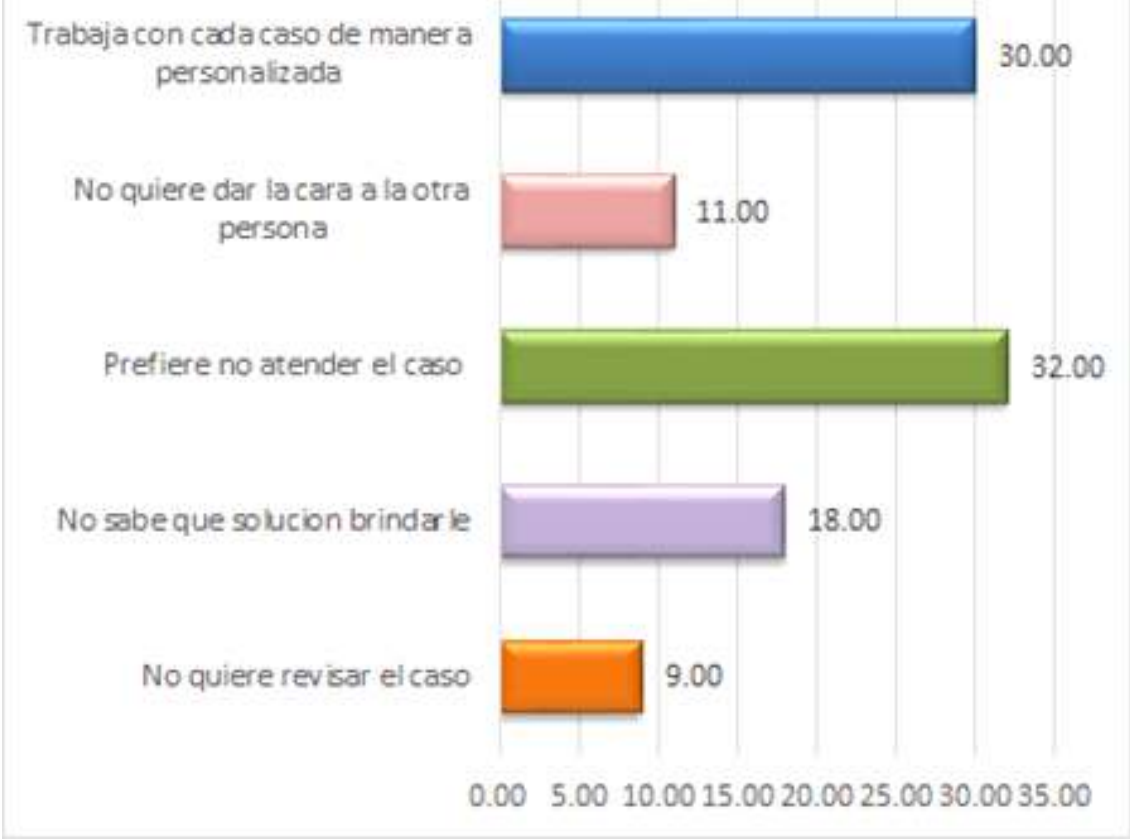

Figura 5. Distribución porcentual de los trabajadores sociales del sector justicia según los efectos de la traumatización vicaria. 
Por todo lo anteriormente descrito se concluye que la traumatización vicaria afecta a los Trabajadores Sociales del sector justicia por el alto número de casos que se le presentan donde la acción profesional resulta imprescindible puesto que con el informe de valor social se toman muchas decisiones que afecta al usuario y/o su familia.

\section{CONCLUSIONES}

1) El factor personal de mayor incidencia presentado por los Trabajadores Sociales del sector Justicia es la traumatización vicaria, la cual se ve traducida por la conducta del profesional por no continuar con el tratamiento del caso, huir del caso y derivar el caso a una instancia mayor o a otro especialista.

2) La traumatización vicaria se manifiesta con llanto, ansiedad, culpa, arrepentimiento, los cuales dificultan la labor del profesional, que se involucra, dejando de lado lo objetivo y priorizando la subjetividad.

3) Los Trabajadores Sociales del sector justicia han presentado traumatización vicaria, en algún estadio de su vida, y han optado por no continuar con el tratamiento hasta el final, por no reconocerlo como tal.

4) La traumatización vicaria dificulta la labor del profesional en el tratamiento de casos sociales, relacionándolo de alguna forma con un episodio de su vida, que se convierte en una situación no resuelta.

\section{REFERENCIAS BIBLIOGRÁFICAS}

Alday, María, et al (2004). El Trabajo Social en el servicio de Justicia. Argentina. Editorial Espacio. Cantu, Carlos (1999). El Trabajo en las agencias sociales. México.

Genolet, Alicia, et al (2005). La profesión del Trabajo Social icosa de mujeres? Argentina. Editorial Espacio.

Kisnerman, Natalio (1989). Pensar el Trabajo Social: Una introducción desde el construccionismo. Argentina. Editorial Lumen Humanitas.

Packman, Marcelo (1997). La psicoterapia en contextos de pobreza. España. Editorial. Gedisa Editorial.

Rosas, Margarita (1998). Una perspectiva teórica metodológica de la intervención en Trabajo Social. Buenos Aires. Editorial Espacios. 